\title{
BEE : Concepteurs d'écosystèmes éducatifs
}

\author{
BEE : Builders of Educative Ecosystem
}

\section{BEE : Constructores de ecosistemas educativos}

\author{
Christophe Laduron, maître de conférence et chercheur \\ Haute École Albert Jacquard de Namur - Université de Liège, Belgique \\ christophe.laduron@heaj.be \\ Isabelle Sacré, maître-assistante \\ Haute École Albert Jacquard de Namur - Centre d'Autoformation et de Formation Continuée, Belgique \\ isabelle.sacre@lecaf.be
}

\begin{abstract}
RÉSUMÉ
BEE porte sur l'accompagnement pédagogique d'équipes d'enseignants, via la construction d'écosystèmes d'apprentissages numériques. L'objectif est d'inverser la relation formateurformé et de l'adapter à l'environnement des futurs enseignants actuels. En soutenant ce changement de paradigme, BEE envisage un apprentissage simultané et une articulation théorie - pratique recourant à la scénarisation de pratiques pédagogiques développant la pensée réflexive. Contenus et dispositifs sont revus en s'aidant d'outils numériques renforçant la formation des futurs enseignants à l'intégration des TICE. BEE met à disposition les ressources techniques, pédagogiques et humaines au service d'une équipe de formateurs désireux de former les futurs enseignants selon les besoins qu'ils manifestent et les ressources dont ils disposent. Cette contribution propose le bilan des deux premières années d'expérimentation du projet.
\end{abstract}

Mots-clés : dispositif hybride, enseignement supérieur, classes inversées, écosystème d'apprentissage, TICE, formation initiale des enseignants

\section{ABSTRACT}

BEE focuses on the pedagogical support of teams of teachers, through the construction of digital learning ecosystems. The objective is to reverse the trainer-trained relationship and 
adapt it to the environment of today's future teachers. By supporting this paradigm shift, BEE is considering simultaneous learning and theory-practice articulation through the scripting of pedagogical practices that develop reflective thinking. Content and devices are reviewed with the help of digital tools to strengthen the training of future teachers in the integration of ICTE. BEE provides technical, pedagogical and human resources to a team of trainers who wish to train future teachers according to their needs and the resources at their disposal. This contribution presents an assessment of the first two years of experimentation of the project.

Keywords: hybrid system, higher education, reverse classes, learning ecosystem, ICTE, initial teacher training

\section{RESUMEN}

BEE se centra en el apoyo pedagógico de equipos de profesores, a través de la construcción de ecosistemas digitales de aprendizaje. El objetivo es invertir la relación entre formador y aprendiz y adaptarla al entorno de los futuros profesores de hoy. Al apoyar este cambio de paradigma, BEE contempla el aprendizaje simultáneo y la articulación teoría-práctica a través del guion de prácticas pedagógicas que desarrollan el pensamiento reflexivo. Se revisan los contenidos y dispositivos con la ayuda de herramientas digitales para fortalecer la formación de los futuros profesores en la integración de las TICE. BEE proporciona recursos técnicos, pedagógicos y humanos a un equipo de formadores que desee formar a los futuros profesores en función de sus necesidades y de los recursos de que disponen. Esta contribución presenta una evaluación de los dos primeros años de experimentación del proyecto.

Palabras clave: sistema híbrido, educación superior, clases inversa, ecosistema de aprendizaje, TICE, formación inicial del profesorado

\section{Contexte}

Le projet BEE (Builders of Educative Ecosystem) trouve ses origines dans le cadre de la formation initiale des enseignants de l'enseignement fondamental, en Fédération Wallonie Bruxelles de Belgique (FWB). Cette formation est actuellement assurée par les catégories pédagogiques des Hautes Écoles, sous la forme d'un baccalauréat de 3 ans. Notons qu'une réforme de celle-ci vient d'être promulguée et sera mise en vigueur dès la rentrée 2020, avec comme modification majeure le passage de 3 à 4 années d'étude ainsi qu'une co-organisation obligatoire de la formation entre Hautes Écoles et Universités.

Le projet est né des constats liés à l'évolution du public des futurs enseignants, de ses attentes et de ses besoins. Ces constats, limités dans un premier temps à notre seule perception de formateurs dans notre Haute École pédagogique, ont pu se nuancer grâce à nos détachements respectifs dans un centre de formation continue pour enseignants et dans un service de recherche en technologie de l'éducation. Ces nouvelles missions nous ont permis d'élargir nos analyses et de les confronter à un échantillon plus vaste de la population enseignante. 


\section{Les constats sous forme de besoins}

Les besoins formulés par nos étudiants, futurs enseignants, sont de différents types. "Organisationnels" tout d'abord : les étudiants assument fréquemment un travail ou une charge familiale, en plus des études qu'ils mènent. Certains éprouvent des difficultés à assister aux cours en présentiel selon l'horaire établi (Tricot, 2017). Par ailleurs, ils souhaitent disposer de plus de temps pour poser des questions à leurs formateurs, à différents moments de leurs parcours.

Les besoins "pédagogiques" ensuite : les étudiants souhaitent pouvoir disposer d'exemples concrets de bonnes pratiques (dans le sens d'un "enseignement prêt à l'emploi") et réclament un accompagnement différencié de la part du formateur lorsqu'il s'agit d'aborder la phase pratique de leur formation : les stages de terrain.

Parallèlement, les enseignants de Haute École en charge de ces étudiants évoquent quant à eux le manque de temps disponible afin d'assurer l'encadrement des étudiants durant la préparation de ces stages et le besoin ressenti d'accompagnement en petits groupes d'étudiants, notamment pour développer la pratique réflexive. Ces mêmes enseignants regrettent également le manque de cas concrets et témoignages de terrain à analyser pour développer la pratique réflexive de leurs étudiants (et dès lors, passer de "l'enseignant prêt à l'emploi" à un "enseignant à (se) construire" (Degraef, 2012).

Degraef, dans son analyse, interroge également le rapport au savoir transmis par les formateurs. Les témoignages qu'elle a pu recueillir mettent en évidence l'écart entre les modèles pédagogiques enseignés en formation initiale des enseignants et les difficultés de les transposer dans la réalité d'une classe. Les enseignants sont à la recherche d'une alternance "théorie-pratique" qui s'articulerait avec les besoins organisationnels cités par les étudiants.

Le projet "BEE" souhaite répondre à ces demandes et privilégier un apprentissage simultané en vue de rompre avec le modèle successif de la formation d'enseignants, pointé notamment lors de l'audit réalisé par l'Agence Européenne pour la Qualité de l'Enseignement Supérieur [AEQES] dans les catégories pédagogiques des Hautes Écoles de la Fédération Wallonie-Bruxelles de Belgique en 2014.

C'est notamment par le biais des TICE (technologies de l'information et de la communication pour l'enseignement) que ce modèle simultané désigné par l'AEQES (2014) pourrait se mettre en place dans le dispositif, au travers de l'analyse de pratiques de classe. Les premières enquêtes réalisées auprès des étudiants confortent cet aspect du dispositif (cfr infra). Par ailleurs, l'intégration des TICE étant privilégiée pour répondre aux premiers besoins exprimés, elle devenait également un nouvel objet d'évaluation, concernant l'utilisation et la familiarisation du public étudiant à ses usages.

Ces constats, observations et évaluations conduisent tous vers une évidence : la nécessité d'envisager et d'amorcer un changement de paradigme au niveau de l'enseignement supérieur, certains modèles pédagogiques privilégiés semblant ne plus être en adéquation avec les besoins et les attentes des étudiants et formateurs actuels.

\section{Des besoins au projet}

Avec le projet d'écosystème "BEE", l'objectif initial est de privilégier de nouveaux dispositifs d'apprentissage, notamment soutenus par l'essor des potentialités offertes par les outils numériques, rendus de plus en plus accessibles. 
Le principe qui sous-tend la démarche de "BEE" est le suivant : l'apprentissage est une expérience que l'on ne peut pas faire à la place de celui qui apprend (Lebrun, 2007). Nous percevons dès lors notre rôle de formateur d'enseignants comme celui d'un accompagnateur dont la fonction est d'aider à la construction du sens dans ce qu'apprend l'étudiant, des connexions qu'il peut établir entre les différents concepts pédagogiques qu'il développe et découvre. La première de nos missions est de lui permettre de s'approprier ces connexions, d'avoir conscience des stratégies qu'il mobilise dans son apprentissage. Pour Jenkins (cité par Berlinguer, 2014) : "l'enseignant devient un " facilitateur » de connaissances entre la quantité d'informations provenant des ressources informatiques et la capacité cognitive de l'étudiant ».

Nous aspirons à ce que le travail de conception que nous réalisons pour rendre transparentes les stratégies mobilisées chez l'apprenant puisse se répercuter dans les futures pratiques de celui-ci, par isomorphisme pédagogique, rendant lui-même transparentes ses stratégies d'apprentissage pour ses futurs élèves. II est également question d'isomorphisme quant à l'intégration des TICE dans les dispositifs d'apprentissage, dont nous observons que le transfert dans la pratique une fois diplômés reste difficile à l'heure actuelle (Delacharlerie, Fiévez, Lennertz et Lumen, 2018). En effet, les usages pédagogiques du numérique restent encore peu fréquents en FWB. Le sentiment de compétence affiché par les enseignants reste interpellant : $57 \%$ affirment "se débrouiller", $14 \%$ n'avoir "aucune connaissance" et $23 \%$ n'ont encore suivi aucune formation en la matière (Delacharlerie et al., 2018).

Si les caractéristiques du dispositif BEE (voir infra) permettent de le qualifier d'innovation pédagogique (Cros, 1997), que ce dernier puisse se positionner tant au niveau de l'innovation de service, pédagogique ou technique (Charlier, Bonamy et Saunders, 2002), se pose également la question de l'engagement et de l'enrôlement des acteurs impliqués directement ou indirectement dans le projet. Comment faire en sorte que cette innovation se partage ? Les réactions, qu'elles proviennent des étudiants bénéficiaires ou des professeurs impliqués, sont de tout ordre et vont de l'adhésion au rejet, sans doute notamment par manque de connaissance du dispositif en tant que tel.

Au-delà de l'évidence de notre volonté (Cros, 1997) à voir proposer ce dispositif comme une option de méthodologie d'apprentissage de plus à l'arc des formateurs d'enseignants, de faire que celui-ci apporte une plus-value à la formation des futurs enseignants, notre hypothèse repose sur l'efficacité accrue d'un modèle de type "accompagnement" à celui d'une formation "classique" en vue d'outiller et former les formateurs désireux d'intégrer le processus.

\section{Caractéristiques principales de BEE}

Les objectifs de notre projet sont donc les suivants :

- soutenir un changement de paradigme qui envisage un apprentissage simultané et une meilleure articulation théorie - pratique, en recourant notamment à la scénarisation de pratiques pédagogiques;

- revoir contenus et dispositifs en s'aidant d'outils numériques qui sont mobilisés par les formateurs d'enseignants, porteurs des écosystème mis en place. II s'agit également, par isomorphisme pédagogique, de renforcer la formation des futurs enseignants à l'intégration des TICE, les compétences des formés étant liées à celles de leur formateur (Roland et Vanmeerhaeghe, 2016);

- s'engager dans la responsabilité collective en co-construisant ses modules de formation, et en développant la co-formation entre maîtres-assistants (formateurs d'enseignants).

Nous nous employons à proposer des modules d'apprentissage au sein de la formation initiale des enseignants, conçus en équipes disciplinaires ou pluridisciplinaires, afin de mieux préparer les étudiants à transférer les notions pédagogiques et didactiques au terrain professionnel. Ces modules sont conçus dans une logique d'intégration et d'apprentissage simultané. 
Une étude réalisée par l'équipe européenne de recherche Eurydice27 met à jour deux modèles de formation différents dans la formation initiale des enseignants du secondaire inférieur en Europe. Le modèle successif fait se dérouler d'abord la totalité de la formation théorique, suivie seulement, ensuite, de la formation pratique. Le modèle simultané propose aux étudiants formation théorique et formation pratique en parallèle. La Belgique francophone a clairement choisi le modèle simultané pour la formation de ses instituteurs primaires (AEQES, 2014).

Par ailleurs, ces modules d'apprentissage, qui ont pour vocation une transposition didactique dans le futur professionnel des étudiants, reposent sur des "écosystèmes d'apprentissages numériques", inspirés des théories relatives aux environnements personnels d'apprentissage (EPA) (Roland et Talbot, 2014). Accompagner les étudiants au sein d'environnement numériques déjà existants leur permettra par ailleurs de développer également les compétences nécessaires à la gestion de leur EPA, compétences qui leur font toujours défaut dans la perspective d'une gestion efficace de ces derniers (Dabbagh et Kitsantas, 2012).

Nous avons décidé de rendre explicites les liens que l'on peut tisser entre l'apprenant et l'objet d'apprentissage au sein d'un écosystème d'apprentissage reposant sur cinq "connecteurs" :

1) Observer / Discerner : observer des exemples de pratique professionnelle pour discerner les caractéristiques pertinentes.

2) Abstraire / conceptualiser : abstraire le concept étudié au départ des caractéristiques pertinentes observées. Construire le concept en confrontant ses idées avec celles de ses pairs.

3) Pratiquer / Partager : s'essayer à une pratique guidée et partager ses essais, ses ressources ou celles qui ont été mises à disposition.

4) Co-construire / Transférer : recontextualiser de façon collective l'apprentissage en co-créant sa propre réalisation.

5) S'évaluer / Se situer : au travers de l'ensemble des phases de l'apprentissage, être accompagné dans son parcours pour développer sa métacognition.

Nous avons établi ces connecteurs comme des couples d'action, en nous inspirant de différents pédagogues. La principale référence utilisée est la méthodologie inductive de Barth (2013), qui propose de mettre l'accent sur la construction du concept par l'analyse de la réalité incarnant le concept. C'est pourquoi le connecteur "Observer/Discerner" regroupe des exemples organisés de pratiques contextualisées (par le biais de vidéos, d'extraits de séquence didactique, ou de témoignages enregistrés) qui deviennent les objets d'analyse pour les étudiants. La tâche de l'étudiant est de faire des liens entre les documents proposés et d'en dégager des éléments communs et pertinents, comme le propose Barth. La mise en commun de ces caractéristiques relevées se déroule en présentiel, de manière à ce que les apprenants puissent confronter leurs avis. Nous nous attardons volontairement sur ce connecteur car il représente la porte d'entrée de notre dispositif. Les quatre autres connecteurs sont tout aussi importants, mais ceux-ci s'organisent de façon plus individualisée. En effet, après avoir découvert en contexte le concept à appréhender, l'étudiant utilise les autres connecteurs en fonction de ses besoins et des tâches qui lui sont demandées. L'ensemble des connecteurs rassemble les différents volets taxonomiques décrits par Bloom, Engelhart, Furst, Hill, et Krathwohl en 1956 (cité par Krathwohl, 2002). 
Ces connecteurs organisent l'écosystème d'apprentissage ainsi que l'environnement numérique proposé à l'étudiant. Ils se composent d'exemples de pratiques professionnelles, de supports d'aide à l'analyse, de ressources théoriques, d'auto-évaluations formatives, d'exercices d'appropriation des notions théoriques abordées (dans différents formats), etc. le tout dans un modèle d'organisation hybride alternant séquences d'apprentissage en présentiel et module d'enseignement à distance. Cette organisation n'est pas sans rappeler celles des "classes inversées" (Lebrun, 2015) et plus spécifiquement dans notre cas de figure, celle de type 3 où se succèdent des phases de recherche et d'analyse à distance, suivies de partages d'informations et d'analyse réflexive en présentiel, qui donnent lieu à la structuration théorique à distance pour enfin aboutir aux phases d'exercisation en présence de l'enseignant. Tout au long du dispositif, les méthodologies inductives (Barth, 2013) sont utilisées afin notamment d'ancrer en permanence les contenus abordés à la pratique de terrain vécue par les étudiants.

Ce type de dispositif permet de dégager de la disponibilité pour travailler en présentiel ces stratégies d'appropriation. Les moments en présentiel sont alors consacrés à rendre explicites les questionnements de chacun, les confrontations d'idées, de présupposés, les partage d'avis ou les constructions de sens propres à chaque apprenant.

Pour construire ces écosystèmes, nous nous sommes constitués une équipe pluridisciplinaire dénommée "BEELABS" (www.beelabs.be). Composée de deux ingénieurs pédagogiques spécialisés en scénarisation pédagogique et en intégration des TICE ainsi que d'un infographiste-réalisateur audio-visuel, elle réunit chaque année des groupes de formateurs d'enseignants (maîtres-assistants ou maîtres de formation pratique) autour de la table, peu importe leurs compétences initiales dans le domaine des TICE. Son but est d'accompagner l'équipe d'enseignants ainsi formée dans la conception de leur écosystème d'apprentissage, en leur fournissant l'accompagnement pédagogique, technologique et médiatique (BEELABS, 2017).

Le travail en équipe est largement privilégié chez ces formateurs, qui apparaît être un moyen pertinent afin de rompre l'isolement de l'enseignant au sein de son unité d'enseignement et d'améliorer l'efficacité de sa pratique. Nous souhaitons que les enseignants s'inscrivent sur base volontaire, avec l'accord de leur direction, et nous privilégions les équipes qui ont déterminé un contenu d'apprentissage commun. Le projet nécessite également que les enseignants se forment en continu aux outils numériques, et est valorisé en tant que formation continuée des enseignants. Une attestation de participation est délivrée au terme du projet.

L'écosystème réalisé permettra notamment de diffuser les différents supports conçus spécifiquement pour l'apprentissage visé. L'écosystème prendra par exemple la forme d'un site internet (ou d'un cloud) conçu et géré par les enseignants impliqués (exemple : www.beepedaprojet.com), au sein duquel l'étudiant se verra proposer des activités de différents types visant le développement de l'apprentissage visé par l'écosystème, des ressources diverses, des liens vers des outils spécifiques et adaptés au contexte d'apprentissages dans lequel ils évoluent, ou encore des tutoriels afin de favoriser le recours à ces derniers une fois sur le terrain.

\section{Bilan critique et transfert}

Au terme de deux années de réflexion, de mise en chantier, d'essais et d'erreurs, identifier les facteurs facilitants et bloquants liés à l'implémentation de ce projet s'avère être l'étape suivante et incontournable, au risque de le voir limité à un stade toujours embryonnaire. 
De ce retour de terrain, nous avons pu dans un premier temps valider notre positionnement en tant que dispositif d'apprentissage hybride. Si nous nous référençons à la typologie Hy-Sup (Burton et al., 2011), BEE correspond à l'écosystème (type 6) et ce en obtenant un score de positionnement de 99,9\%. Ce positionnement nous permet donc de cibler les forces d'un tel dispositif mais nous avertit également des revers dont nous devons nous méfier.

Dans une perspective similaire, nous avons comparé les caractéristiques de notre dispositif BEE au système d'instruments définit par Bourmaud (2007). Une nouvelle fois, cette confrontation a pu renforcer les forces et les piliers sur lesquels nous nous sommes basés. Nous positionner garantit ainsi le maintien d'un certain cap, et évite le risque de perdre de vue certaines de caractéristiques originelles du dispositif.

En conclusion, nous pouvons affirmer que les dispositifs de type BEE répondent aux besoins que nous avions identifiés dans le sens où ils organisent un ensemble d'instruments et de ressources hétérogènes et ils reposent sur des phases d'apprentissage complémentaires mais également redondantes de par leur disponibilité continue pour les étudiants; ils permettent d'envisager plus aisément la différenciation des phases d'apprentissage d'un étudiant à l'autre.

L'instrument pivot, organisateur de ceux qui gravitent autour (Bourmaud, 2007), qui se concrétise par le cœur de l'écosystème BEE dans chacune de ses éditions, est également identifié comme étant une condition nécessaire si l'on souhaite répondre aux besoins évoqués en amont.

A ce jour, nous étudions principalement jusqu'à quel point l'écosystème d'apprentissage numérique organisé tel que nous le concevons, autour de son instrument pivot, permet de tisser le lien entre le futur enseignant et l'ensemble des savoirs et savoir-faire pédagogiques inhérents à son futur métier, et ainsi favoriser un transfert plus efficace à sa pratique.

Depuis sa seconde année d'existence, notre travail ne se limite plus à l'enseignement supérieur. Nous proposons également des formations continues de trois jours destinées aux enseignants de l'enseignement secondaire, répartis en groupes de travail disciplinaires ou interdisciplinaires, désireux d'approfondir leurs connaissances à propos des classes inversées, en vue pour certains d'entre eux de la mettre en œuvre dans leur pratique.

Ces modules de formation continue ont déjà fait l'objet d'une première phase d'évaluation (par questionnaire) et de régulation. Celle-ci nous a déjà permis de constater que les freins observés à l'entrée chez les étudiants bénéficiaires de ce type de dispositif restent relativement identiques à ceux rencontrés par les enseignants. Nous pensons notamment au faible niveau initial des bénéficiaires de ces formations en termes de compétences numériques, induisant inévitablement chez eux un sentiment de compétence relativement faible pour l'intégration des TICE dans leur pratique pédagogique (ne faisant que confirmer les observations effectuées dans le cadre du baromètre Digital Wallonia 2018 (Delacharlerie et al., 2018)). Les enseignants ont également confirmé la déstabilisation qu'ils ont vécue suite à l'évolution de leur rapport au savoir et à la gestion de la classe, deux bouleversements induits par le changement de posture inévitable lié à la mise en place des classes inversées de type 2 et 3 (Lebrun, Lison et Batier, 2016).

A ce stade, nous ne sommes pas encore en mesure d'évaluer avec rigueur l'impact du dispositif BEE sur les étudiants, et encore moins sur les enseignants accompagnés dans la construction de leur dispositif. Nous avons pu néanmoins fournir une conclusion provisoire concernant l'impact positif du premier dispositif, portant sur la pédagogie par projet, qui a d'ailleurs été reprogrammé par les enseignants concepteurs à deux reprises, et administré par eux de façon autonome.

Le suivi des projets en cours nous permettra de voir dans quelle mesure les pratiques d'enseignement intègrent ce dispositif BEE de façon pérenne, ainsi que dans le transfert à d'autres contenus d'apprentissage. 
Les principaux apports que nous identifions à ce jour, et qui devront faire l'objet d'analyses spécifiques au travers des futures éditions des écosystèmes $B E E$, représentent autant de pistes à mettre en regard de l'évolution indispensable des approches méthodologiques dans l'enseignement supérieur et plus spécifiquement dans la formation initiale des enseignants. Selon nous, ils portent prioritairement sur :

- la construction des connaissances et des compétences dans l'enseignement supérieur pédagogique;

- la construction, la gestion et la régulation des environnements d'apprentissages;

- l'impact - l'efficience - la perception de l'efficacité des classes inversées dans l'enseignement supérieur;

- les usages pédagogiques de la vidéo dans l'enseignement supérieur;

- l'isomorphisme techno-pédagogique dans la formation initiale à l'intégration des TICE;

- la posture du formateur d'enseignant dans des dispositifs d'apprentissage hybrides.

\section{Liste de références}

Agence pour l'Évaluation de la Qualité de l'Enseignement Supérieur. (2014). Évaluation du cursus INSTITUTEUR(-trice) PRIMAIRE en Fédération Wallonie-Bruxelles ANALYSE TRANSVERSALE.

Barth, B.-M. (2013). L'apprentissage de l'abstraction. Paris: Retz. Repéré à http://www.editionsretz.com/pedagogie/l-apprentissage-de-l-abstraction-9782725632438.html

BEELABS. (2017). Mission, histoire et services de BEELABS. Repéré à, https://www.beelabs.be/beelabs

Berlinguer, L. (2014). L'enseignant dans l'école de l'autonomie. Administration et éducation: Revue de l'association française des administrateurs de l'éducation, 144(4), 15-21.

Bourmaud, G. (2007). L'organisation systémique des instruments : méthodes d'analyse, propriétés et perspectives de conception ouvertes. Dans Colloque de l'Association pour la Recherche Cognitive ARCo'07 : Cognition - Complexité Collectif, 61-76. Nancy. Repéré à https://hal.inria.fr/inria-00191128

Burton, R., Deschryver, N., Lebrun, M., Charlier, B., Docq, F., Borruat, S., ... Villiot-Leclercq, E. (2011). Vers une typologie des dispositifs hybrides de formation en enseignement supérieur. Distances et Savoirs, 9(1), 69-96. https://doi.org/10.3166/ds.9.69-96

Charlier, B., Bonamy, J., et Saunders, M. (2002). Apprivoiser l'innovation. In B. Charlier, \& D. Peraya (Eds.), Apprendre les technologies pour l'éducation : analyses de cas, théories de référence, guides pour l'action (p. 45-67). Brussels: De Boeck.

Cros, F. (1997). L'innovation en éducation et en formation. Revue Française de Pédagogie, 1997(118), $127-$ 156.

Dabbagh, N. et Kitsantas, A. (2012). Personal Learning Environments, social media, and self- regulated learning: A natural formula for connecting formal and informal learning. The Internet and Higher Education, 15(1), 3-8.

Degraef, V. (2012). Évaluation qualitative, participative et prospective de la formation intiale des enseignants en Fédération Wallonie-Bruxelles. Repéré à : http://enseignement.be/download.php?do id=9998

Delacharlerie, A., Fiévez, A., Lennertz, S., et Lumen, J. (2018). Baromètre Digital Wallonia Education \& Numérique 2018. Jambes, Belgique.

https://content.digitalwallonia.be/post/20180322084629/Baromètre-2018-Digital-Wallonia-EducationNumérique.pdf

Krathwohl, D. R. (2002). A revision of Bloom's Taxonomy: An Overview. Theory into Pratice, 41(4), 302. https://doi.org/10.1207/s15430421tip4104 2 
Lebrun, M. (2007). Théories et méthodes pédagogiques pour enseigner et apprendre: Quelle place pour les TIC dans l'éducation? De Boeck Supérieur. https://doi.org/10.3917/dbu.lebru.2007.02

Lebrun, M., et Lecoq, J. (2015). Classes inversées Enseigner et Apprendre à l'endroit. Réseau Canopé.

Lebrun, M., Lison, C., et Batier, C. (2016). Les effets de l'accompagnement technopédagogique des enseignants sur leurs options pédagogiques, leurs pratiques et leur développement professionnel. Revue Internationale de Pédagogie de l'Enseignement Supérieur, 32(1), 1-20. Repéré à : http://ripes.revues.org/1028

Roland, N., et Talbot, L. (2014). L'environnement personnel d'apprentissage : un système hybride d'instruments. Sticef, 2014(21), 289-316.

Roland, N., et Vanmeerhaeghe, S. (2016). Les formateurs d'enseignants face aux environnements personnels d'apprentissage de leurs étudiants : représentations et accompagnement. Revue internationale de pédagogie de I' enseignement supérieur, 32(1), 1-20.

Tricot, A. (2017). L'innovation pédagogique. Paris: Retz 